\title{
Determination and Smoothing of Fourier Coefficients Representing Piecewise Continuous Functions
}

\author{
B. A. Peavy \\ Institute for Applied Technology, National Bureau of Standards, Washington, D.C. 20234
}

(February 7, 1967)

\begin{abstract}
This paper presents a method of solving for Fourier coefficients where the dependent variable can be expressed as a piecewise continuous function, when various conditions of continuity and smoothing are assumed. An example is included to show the effect of smoothing in the region of a discontinuity for a system composed of two materials that exhibit a discontinuity at their interface and surrounded by a third material which does not have a discontinuity. An advantage to be gained from smoothing is an increase in the convergence of a finite Fourier series representation of a piecewise continuous function in the region of the discontinuities.
\end{abstract}

Key Words: Continuous function, Fourier series, piecewise, smoothing.

\section{Introduction}

Many time-independent problems of a practical nature involve the solution to the partial differential equation

$$
\frac{\partial^{2} v}{\partial x^{2}}+\frac{\partial^{2} v}{\partial y^{2}}+\frac{\partial^{2} v}{\partial z^{2}}=0
$$

in rectangular coordinates, or

$$
\frac{\partial^{2} v}{\partial r^{2}}+\frac{1}{r} \frac{\partial v}{\partial r}+\frac{1}{r^{2}} \frac{\partial^{2} v}{\partial \phi^{2}}+\frac{\partial^{2} v}{\partial z^{2}}=0
$$

in cylindrical coordinates. From the general solution, one particular solution is in the form

$$
v=B+C z+\sum_{n} A_{n} \sin \frac{n \pi z}{\omega},
$$

where the Fourier coefficients, $A_{n}=A_{n}(x, y)$ for rectangular coordinates, or $A_{n}=A_{n}(r, \varphi)$ for cylindrical coordinates, are determined from boundary conditions in the region $0 \leqslant z \leqslant \omega$, and fixed coordinate positions for $x$ and $y$, or $r$ and $\varphi$, with the condition that other necessary solutions of (1) disappear on this boundary. Also, $B+C z$ must satisfy $v$ at the end points, $z=0$ and $z=\omega$.

From an experimental point of view, the dependent variable, $v$, can be approximated from measured values at several $z$ positions on the boundary by a relationship, $v=g(z)$. The Fourier coefficients are usually simply determined if the function $g(z)$ and its derivatives are continuous over the region $0 \leqslant z \leqslant \omega$. For various applications, the variable $v$ must be expressed as piecewise continuous functions in this region, with apparent discontinuities at discrete points. If it is known that the discontinuities do not exist, then an appropriate smoothing function can be employed.

The purpose of this paper is to solve for Fourier coefficients, when it is assumed that the dependent variable $v$ can be expressed by polynomials representing discrete portions of the region $0 \leqslant z \leqslant \omega$, and that conditions of continuity and smoothing exist. An application of functions developed to a hypothetical problem is also presented. 


\section{Analysis}

On a fixed surface lying in the region $0 \leqslant z \leqslant \omega$, the dependent variable $v$ is defined

$$
v=g_{i}(z) ; \quad z_{i-1}<z<z_{i},
$$

where $i=1,2,3, \ldots m, z_{0}=0$, and $z_{m}=\omega$. The functions $g_{i}(z)$ and their derivatives may have discontinuities at the points $z_{i}, i=1,2, \ldots m-1$. Substituting in (2), multiplying through by $\sin k \pi z / \omega$, and integrating between the specified limits gives the following equations

$$
\int_{z_{i-1}}^{z_{i}}\left[g_{i}(z)-B-C z\right] \sin \frac{k \pi z}{\omega} d z=\sum_{n} A_{n} \int_{z_{i-1}}^{z_{i}} \sin \frac{n \pi z}{\omega} \sin \frac{k \pi z}{\omega} d z
$$

Adding the above equations

$$
\sum_{n} A_{n} \int_{0}^{\omega} \sin \frac{n \pi z}{\omega} \sin \frac{k \pi z}{\omega} d z=-\int_{0}^{\omega}(B+C z) \sin \frac{k \pi z}{\omega} d z+\sum_{i=1}^{m} \int_{z_{i-1}}^{z_{i}} g_{i}(z) \sin \frac{k \pi z}{\omega} d z
$$

from which

$$
A_{n}=\frac{2}{n \pi}\left\{C \omega(-1)^{n}-B\left[1-(-1)^{n}\right]\right\}+\frac{2}{\omega} \sum_{i=1}^{m} \int_{z_{i-1}}^{z_{i}} g_{i}(z) \sin \frac{n \pi z}{\omega} d z
$$

Repeated integration by parts yields

$$
\begin{aligned}
\int g_{i}(z) \sin \frac{n \pi z}{\omega} d z=\frac{\sin \frac{n \pi z}{\omega}}{\left(\frac{n \pi}{\omega}\right)^{2}}\left\{g_{i}^{\prime}(z)-\frac{g_{i}^{\prime \prime \prime}(z)}{\left(\frac{n \pi}{\omega}\right)^{2}}+\frac{g_{i}^{\mathrm{v}}(z)}{\left(\frac{n \pi}{\omega}\right)^{4}}-\ldots\right\} \\
\quad-\frac{\cos \frac{n \pi z}{\omega}}{\frac{n \pi}{\omega}}\left\{g_{i}(z)-\frac{g_{i}^{\prime \prime}(z)}{\left(\frac{n \pi}{\omega}\right)^{2}}+\frac{g_{i}^{\mathrm{jv}}(z)}{\left(\frac{n \pi}{\omega}\right)^{4}}-\ldots\right\},
\end{aligned}
$$

where superscripts ', "', "', represent the first, second, third, etc., derivative with respect to $z$. Letting

$$
\begin{gathered}
G_{i}=\frac{\sin \frac{n \pi z_{i}}{\omega}}{\left(\frac{n \pi}{\omega}\right)^{2}}\left\{g_{i}^{\prime}\left(z_{i}\right)-g_{i+1}^{\prime}\left(z_{i}\right)-\frac{\left[g_{i}^{\prime \prime \prime}\left(z_{i}\right)-g_{i+1}^{\prime \prime \prime}\left(z_{i}\right)\right]}{\left(\frac{n \pi}{\omega}\right)^{2}}+\ldots . .\right\} \\
-\frac{\cos \frac{n \pi z_{i}}{\omega}}{\frac{n \pi}{\omega}}\left\{g_{i}\left(z_{i}\right)-g_{i+1}\left(z_{i}\right)-\frac{\left[g_{i}^{\prime \prime}\left(z_{i}\right)-g_{i+1}^{\prime \prime}\left(z_{i}\right)\right]}{\left(\frac{n \pi}{\omega}\right)^{2}}+\ldots .\right\}, \\
H=\frac{g_{1}^{\prime \prime}(0)}{\left(\frac{n \pi}{\omega}\right)^{2}}-\frac{g_{1}^{i v}(0)}{\left(\frac{n \pi}{\omega}\right)^{4}}+\ldots . \\
K=\frac{g_{m}^{\prime \prime}(\omega)}{\left(\frac{n \pi}{\omega}\right)^{2}}-\frac{g_{m}^{\mathrm{iv}}(\omega)}{\left(\frac{n \pi}{\omega}\right)^{4}}+\ldots .
\end{gathered}
$$


then

$$
A_{n}=\frac{2}{n \pi}\left\{g_{i}(0)-B+(-1)^{n}\left[C \omega+B-g_{m}(\omega)\right]-H+(-1)^{n} K\right\}+\frac{2}{\omega} \sum_{i=1}^{m-1} G_{i} .
$$

For the condition that $B=g_{1}(0)$ and $C \omega+B=g_{m}(\omega)$, the Fourier coefficients are

$$
A_{n}=\frac{2}{n \pi}\left\{(-1)^{n} K-H\right\}+\frac{2}{\omega} \sum_{i=1}^{m-1} G_{i} .
$$

Assume for the region, $0 \leqslant z \leqslant \omega$, that $v$ as a function of $z$ behaves as shown in figure 1, where $g_{1}, g_{2}, g_{3}$, and $g_{4}$ are known functions of $v$, (perhaps as determined from experiment) and $f_{1}, f_{2}$, and $f_{3}$ encompass regions of uncertainty or regions which cannot be defined by experiment. If it is known that there are no discontinuities within the regions of the uncertainties, it is possible to smooth or bridge between the known functions with a cubic polynomial which gives continuity to two adjacent $g$ functions and their first derivatives. Examining the first region that includes an uncertainty, the cubic polynomial, $f_{1}(z)=M_{0}+M_{1} z+M_{2} z^{2}+M_{3} z^{3}$ is solved for the coefficients, $M$, such that $\mathrm{g}_{1}\left(z_{1}\right)=f_{1}\left(z_{1}\right), g_{1}^{\prime}\left(z_{1}\right)=f_{1}^{\prime}\left(z_{1}\right), g_{2}\left(z_{2}\right)=f_{1}\left(z_{2}\right)$ and $g_{2}^{\prime}\left(z_{2}\right)=f_{1}^{\prime}\left(z_{2}\right)$, from which the necessary coefficients are

$$
\begin{aligned}
& M_{2}=\frac{3\left[g_{2}\left(z_{2}\right)-g_{1}\left(z_{1}\right)\right] \bar{z}-\epsilon\left[(3 \bar{z}+\epsilon) g_{1}^{\prime}\left(z_{1}\right)+(3 \bar{z}-\epsilon) g_{2}^{\prime}\left(z_{2}\right)\right]}{4 \epsilon^{3}} \\
& M_{3}=\frac{\epsilon\left[g_{1}^{\prime}\left(z_{1}\right)+g_{2}^{\prime}\left(z_{2}\right)\right]+g_{1}\left(z_{1}\right)-g_{2}\left(z_{2}\right)}{4 \epsilon^{3}},
\end{aligned}
$$

where $\bar{z}=z_{1}+\epsilon=z_{2}-\epsilon$ is the midpoint between $z_{1}$ and $z_{2}$. Assuming bridging between $g_{1}$ and $g_{2}$, then

$$
G_{1}=F_{1}+F_{2}
$$

where

$$
\begin{aligned}
& F_{1}=\frac{1}{\left(\frac{n \pi}{\omega}\right)^{4}}\left[\left\{g_{2}^{\prime \prime \prime}\left(z_{2}\right)-\frac{g_{2}^{\mathrm{V}}\left(z_{2}\right)}{\left(\frac{n \pi}{\omega}\right)^{2}}+\ldots\right\} \sin \frac{n \pi z_{2}}{\omega}-\left\{g_{1}^{\prime \prime \prime}\left(z_{1}\right)-\frac{g_{1}^{\mathrm{V}}\left(z_{1}\right)}{\left(\frac{n \pi}{\omega}\right)^{2}}+\ldots\right\} \sin \frac{n \pi z_{1}}{\omega}\right] \\
& \left.\quad+\frac{1}{\left(\frac{n \pi}{\omega}\right)^{3}}\left[g_{1}^{\prime \prime}\left(z_{1}\right)-\frac{g_{1}^{\mathrm{IV}}\left(z_{1}\right)}{\left(\frac{n \pi}{\omega}\right)^{2}}+\ldots\right\} \cos \frac{n \pi z_{1}}{\omega}-\left\{g_{2}^{\prime \prime}\left(z_{2}\right)-\frac{g_{2}^{\mathrm{IV}}\left(z_{2}\right)}{\left(\frac{n \pi}{\omega}\right)^{2}}+\ldots\right\} \cos \frac{n \pi z_{2}}{\omega}\right]
\end{aligned}
$$

FigURE 1. Variation of $\mathrm{v}$ with respect to $\mathrm{z}$ showing the defined functions $\mathrm{g}_{1}, \mathrm{~g}_{2}, \mathrm{~g}_{3}$, and $\mathrm{g}_{4}$ connected by functions $\mathrm{f}_{1}, \mathrm{f}_{2}$, and $\mathrm{f}_{3}$ through regions of uncertainty.

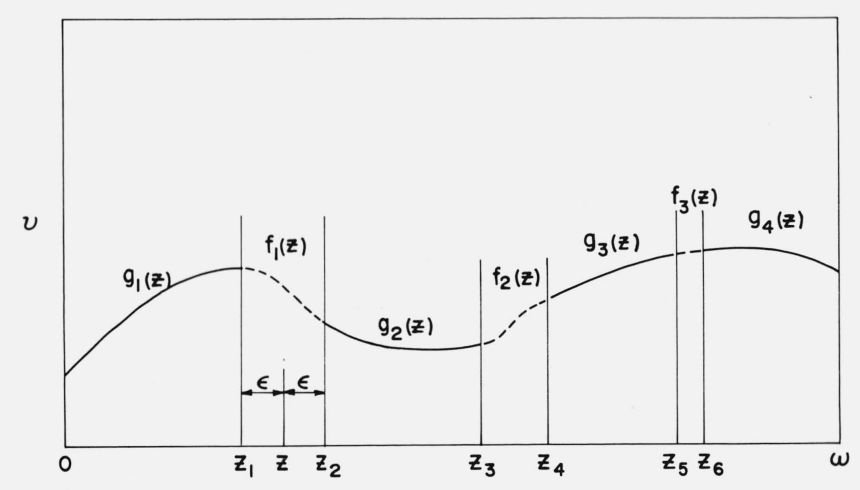


and

$$
\begin{aligned}
F_{2}=\frac{1}{\left(\frac{n \pi}{\omega}\right)^{4}}\left[f_{1}^{\prime \prime \prime}\left(z_{1}\right) \sin \frac{n \pi z_{1}}{\omega}-f_{1}^{\prime \prime \prime}\left(z_{2}\right) \sin \frac{n \pi z_{2}}{\omega}\right] & \\
& +\frac{1}{\left(\frac{n \pi}{\omega}\right)^{3}}\left[f_{1}^{\prime \prime}\left(z_{2}\right) \cos \frac{n \pi z_{2}}{\omega}-f_{1}\left(z_{1}\right) \cos \frac{n \pi z_{1}}{\omega}\right] .
\end{aligned}
$$

Making the substitutions $z_{1}=\bar{z}-\epsilon, z_{2}=\bar{z}+\epsilon, f_{1}^{\prime \prime}(z)=2 M_{2}+6 M_{3} z$, and $f_{1}^{\prime \prime \prime}(z)=6 M_{3}$ in $F_{2}$, yields

$$
\begin{aligned}
F_{2}=\left[g_{1}^{\prime}\left(z_{1}\right)-g_{2}^{\prime}\left(z_{2}\right)\right] & \frac{U\left(\frac{n \pi \epsilon}{\omega}\right) \sin \frac{n \pi \bar{z}}{\omega}}{\left(\frac{n \pi}{\omega}\right)^{2}} \\
& -\left[g_{1}\left(z_{1}\right)-g_{2}\left(z_{2}\right)+\epsilon\left\{g_{1}^{\prime}\left(z_{1}\right)+g_{2}^{\prime}\left(z_{2}\right)\right\}\right] \frac{T\left(\frac{n \pi \epsilon}{\omega}\right) \cos \frac{n \pi \bar{z}}{\omega}}{\frac{n \pi}{\omega}}
\end{aligned}
$$

where

$$
\begin{aligned}
U(\beta) & =\frac{\sin \beta}{\beta}=1-\frac{\beta^{2}}{3 !}+\frac{\beta^{4}}{5 !}-\ldots \\
T(\beta) & =\frac{3}{\beta^{3}}(\sin \beta-\beta \cos \beta) \\
& =3\left(\frac{1}{3 \cdot 1 !}-\frac{\beta^{2}}{5 \cdot 3 !}+\frac{\beta^{4}}{7 \cdot 5 !}-\ldots\right) .
\end{aligned}
$$

Plots of $U(\beta)$ and $T(\beta)$ are given in figure 2 .

For the interval $z_{1} \leqslant z \leqslant z_{2}$, (4b) is the bridging or smoothing function that gives continuity to $g_{1}(z)$ and $g_{2}(z)$ and their first derivatives at $z_{1}$ and $z_{2}$, respectively. This function also eliminates computation of the cubic polynomial $f_{1}(z)$, where the values of $M_{2}$ and $M_{3}$ sometimes give inordinately large values with subsequent loss of significance. The same analysis can be applied to the regions encompassed by $f_{2}(z)$ and $f_{3}(z)$ so that $G_{2}$ and $G_{3}$ can be found.

\section{Specific Cases}

Where successive differentiation terminates, the functions $g_{i}(z)$ may be represented by polynomials. There are many variations of $g_{i}(z)$ possible, but probably the best example is to examine a set of straight lines as given in the following specific cases.

\subsection{Functions as Straight Lines}

The functions $g_{i}(z)$ are represented by a set of straight lines. Here $g_{i}^{\prime \prime}(z)=H=K=0$, so that (3) becomes

$$
A_{n}=\frac{2}{\omega} \sum G_{i}
$$


FIGURE 2. Variation of $\mathrm{U}(\beta)$ and $\mathrm{T}(\beta)$ with $\beta$.

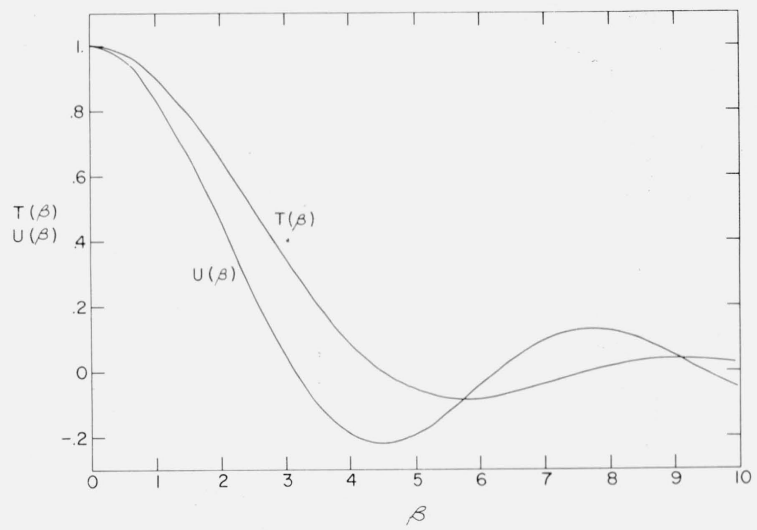

Figure 2. Variation of $\mathrm{U}(\beta)$ and $\mathrm{T}(\beta)$ with $\beta$.

If a set of straight lines which do not intersect at critical positions $z_{i}$, as shown in figure 3 are assumed, then

$$
G_{i}=\left(\frac{\omega}{n \pi}\right)^{2}\left[g_{i}^{\prime}\left(z_{i}\right)-g_{i+1}^{\prime}\left(z_{i}\right)\right] \sin \frac{n \pi z_{i}}{\omega}-\frac{\omega}{n \pi}\left[g_{i}\left(z_{i}\right)-g_{i+1}\left(z_{i}\right)\right] \cos \frac{n \pi z_{i}}{\omega},
$$

where the functions and derivatives are evaluated at $z_{i}$. For the cases where the lines do intersect at $z_{i}$, i.e., $g_{i}=g_{i+1}$, the second term on the right side of (6) is zero.

\subsection{Straight Line Functions Smoothed Near Intersections}

The convergence of (6) when substituted in (5) and (2) is not very satisfactory, and it is possible to increase the convergence by joining the straight lines with smoothing curves that give continuity to the lines and their first derivatives at points $z_{i}-\gamma_{i}$ and $z_{i}+\epsilon_{i}$, as shown in figure 4.

Proceeding as in the derivation of (4), where $F_{1}=0$, yields

$G_{i}=\left[\left(\epsilon_{i}-\gamma_{i}\right)\left(g_{i+1}^{\prime}\left(z_{i}\right)-g_{i}^{\prime}\left(z_{i}\right)\right)+2\left(g_{i+1}\left(z_{i}\right)-g_{i}\left(z_{i}\right)\right)\right] \frac{T\left(n \lambda_{i}\right) \cos \alpha_{i}}{2\left(\frac{n \pi}{\omega}\right)}$

$$
-\left\{g_{i+1}^{\prime}\left(z_{i}\right)-g_{i}^{\prime}\left(z_{i}\right)\right\} \frac{U\left(n \lambda_{i}\right) \sin \alpha_{i}}{\left(\frac{n \pi}{\omega}\right)^{2}}
$$

where

$$
\begin{aligned}
& \alpha_{i}=\frac{n \pi}{2 \omega}\left(2 z_{i}+\epsilon_{i}-\gamma_{i}\right) \\
& \lambda_{i}=\frac{\pi}{2 \omega}\left(\epsilon_{i}+\gamma_{i}\right) .
\end{aligned}
$$

As $\epsilon_{i}=\gamma_{i} \rightarrow 0$, (7) becomes (6). There are many possible specific cases for (7), i.e., $\epsilon_{i}=0$, $\gamma_{i}=0, \epsilon_{i}=\gamma_{i}$, etc. Essentially, smoothing increases convergence by introducing the factors $T(\beta)$ and $U(\beta), \beta=n \lambda_{i}$, to the terms on the right side of (6). The plots $T(\beta)$ and $U(\beta)$ (fig. 2) indicate that the advantage of the increase in convergence is not realized until $\beta \gg 0$. 


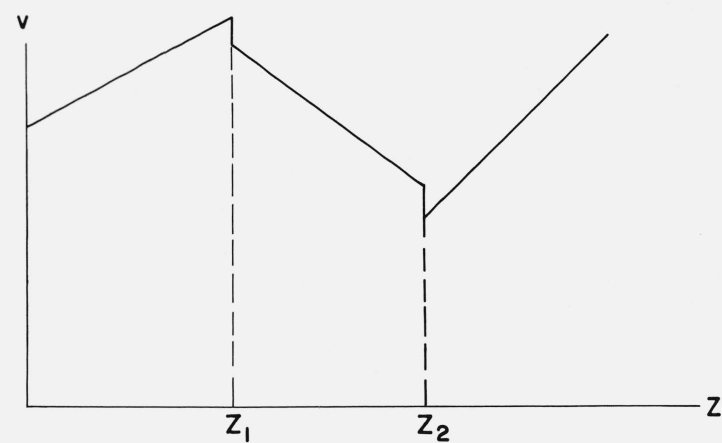

FIgURE 3. Variation of $\mathrm{v}$ as a set of straight lines with function discontinuities at $\mathrm{z}_{1}$ and $\mathrm{z}_{2}$.



FIGURE 4. Variation of $v$ as a set of straight lines with smoothing in the regions of $\mathrm{z}_{1}$ and $\mathrm{z}_{2}$.

\subsection{Example of Smoothing}

Figure 5 is used as an example of the variation of the dependent variable $v$ with the dimension $z$ in order to show the need for smoothing in the region of a discontinuity. For a hypothetical process, consider the potential on the surface of a system of two materials which is surrounded by a third material (not a perfect insulator), and where, on the surface of the system, the region $0 \leqslant z \leqslant 0.5$ represents the potential in a perfect conductor, the region $0.5 \leqslant z \leqslant 1.0$ represents that in a material of finite conductivity, and the discontinuity represents the contact resistance between the two materials. Usually, experimental processes allow measurement of potential in the two regions, but measurements at or near the discontinuity are not always possible or desirable. Assuming homogeneous isotropic materials, measurements taken in the two separate regions give linear relations which, when extrapolated to the point of contact $(z=0.5)$, do not meet in a common point and give an apparent discontinuity. When it is known that the process must be continuous, i.e., the surrounding material is continuous at $z=0.5$, then a smoothing function may be applied.

Numerical values for substitution in (7) are $z_{1}=0.5, g_{1}=1.0, g_{1}^{\prime}=0, g_{2}=0.95, g_{2}^{\prime}=1.9$, and $\epsilon=\gamma$, where $\alpha=n \pi / 2, \lambda=\pi \epsilon$. Substitution in (7), (5) and (2) gives

$$
v=1-z-\sum_{n}(-1)^{n}\left\{\frac{T(2 n \lambda) \sin 2 \pi n z}{20 n \pi}+\frac{3.8 U[(2 n-1) \lambda] \sin (2 n-1) \pi z}{(2 n-1)^{2} \pi^{2}}\right\} .
$$

Figure 6 is a plot of $v$ in the region of the discontinuity shown in figure 5 for the summation of 200 terms of the above series and values of $\epsilon=0.0,0.002,0.003$, and 0.004 , and their respective smoothing curves. Figure 6 shows the influence of the increased convergence of the series toward its

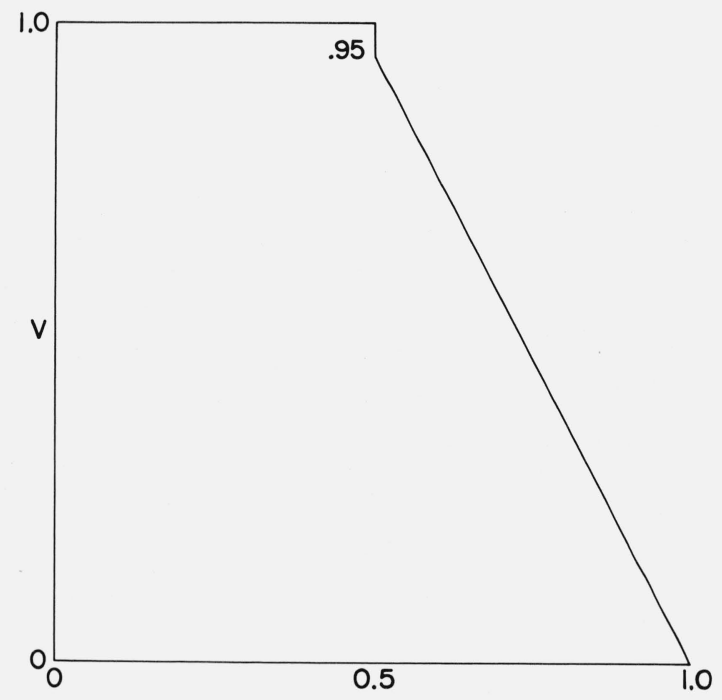

FiguRE 5. Variation of $\mathrm{v}$ with $\mathrm{z}$ for the problem of section 3.3. 
FIGURE 6. Numerical values of $\mathrm{v}$ from (8) in the region of the discontinuity of figure 5 for a summation of 200 terms and values of $\epsilon=0,0.002,0.003$ and 0.004 .

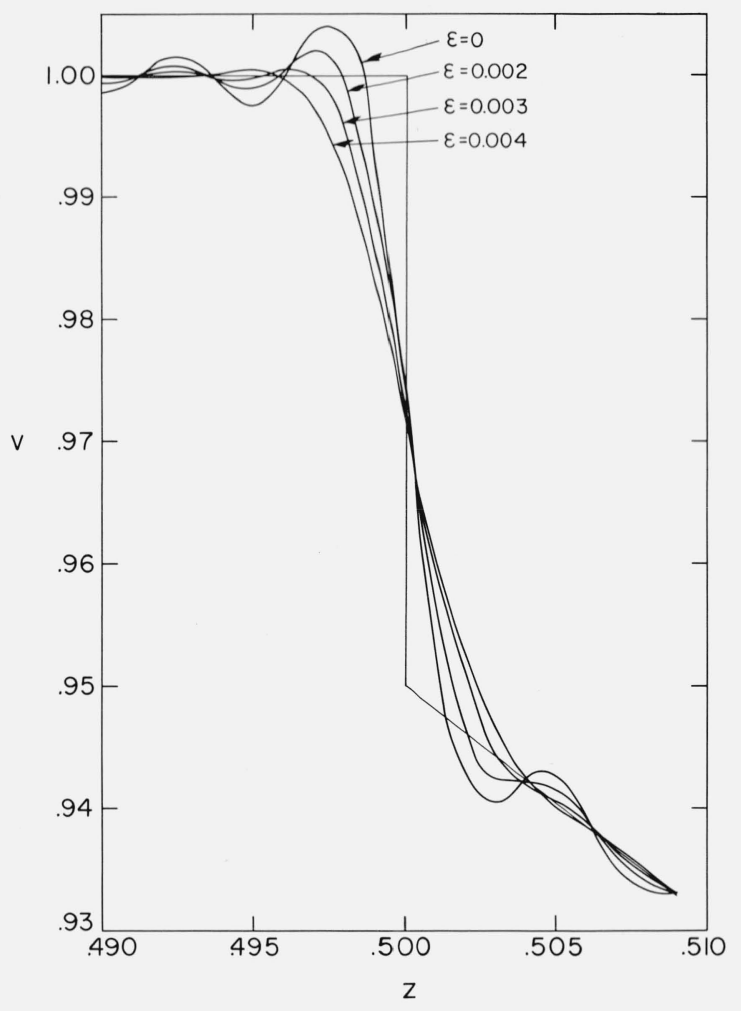

intended values with increase in the value of $\epsilon$. For this case, fairly good convergence of the series is attained for $n>1 / 2 \epsilon$. For the summation of 300 terms, the fluctuations shown on figure 6 are virtually eliminated, except for $\epsilon=0$.

\section{Discussion}

The example of section 3.3 as shown in figure 5 was chosen because it illustrates an extreme condition that a physical process may undergo; that is, the dependent variable has a zero slope up to the discontinuity at the point $z=0.5$. It is then to be expected that actual physical processes where apparent discontinuities occur would give better convergence when smoothing conditions are employed.

Generally, the Fourier sine series (2) can be used to represent a piecewise continuous function over an interval, $0 \leqslant z \leqslant \omega$, by the use of (3). Numerical evaluation will, of course, present problems of convergence in the regions where discontinuities occur (as shown in fig. $6, \epsilon=0$ ). The lack of sufficient convergence in (2) can cause serious problems when (2) is used in the solution of (1). It is therefore expedient to employ the bridging or smoothing relationship (4) (or (7) for the special case of a set of straight lines) for which adequate convergence can be assured for a reasonable number of terms in the series of (2).

A fairly simple heat transfer system that may be analyzed employing the method presented in this paper is shown in figure $7 \mathrm{a}$ by a cross section through a composite cylinder. The inner cylinder (regions 1 and 2) $0 \leqslant r \leqslant a$ is composed of two materials of different thermal conductivities, usually with values greater than that for the material of the outer cylinder (region 3). For the determination of thermal conductivity, region 1 may comprise a material of known thermal conductivity and region 2 a material of thermal conductivity to be determined. It is necessary to determine the heat transfer characteristics in the material of region 3 in order to evaluate the radial heat loss or gain of the inner cylinder, $0 \leqslant r \leqslant a$.

The temperature distribution at $r=a$ will vary according to the thermal conductivities of the materials of regions 1 and 2, and the distribution may have an infinite number of variations. One 


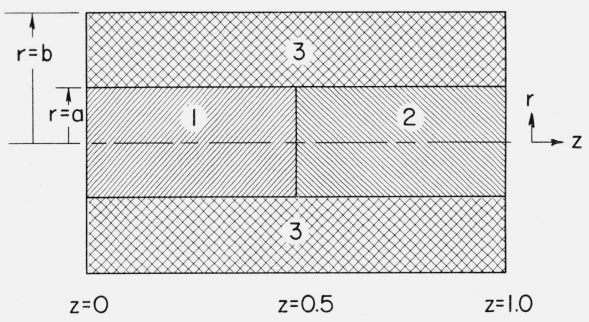

FIGURE 7. Heat transfer system.

(a) Cross-section through composite cylinder.

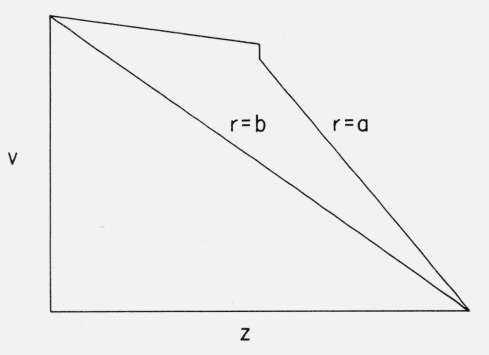

(b) A possible variation in temperature potential. $v$ at $r=a$ and $r=b$.

such distribution for $r=a$ with a thermal contact resistance at $z=0.5$ is shown in figure $7 \mathrm{~b}$, where the intended heat flow is in the positive $z$-direction with a unit temperature potential at $z=0$ and a zero value at $z=1$. On $r=b$ the temperature is assumed to decrease linearly in the above limits. considering region 3 only, a solution of (1) (assuming no $\phi$ dependence) is

$$
v=1-z+\sum \frac{A_{n} F_{0}(n \pi r, n \pi b) \sin n \pi z}{F_{0}(n \pi a, n \pi b)}
$$

where $F_{0}(x, y)=I_{0}(x) K_{0}(y)-I_{0}(y) K_{0}(x)$ and $I_{0}$ and $K_{0}$ are modified Bessel functions of zero order and first and second kind, respectively. The substitution of $r=a$ in (9) gives a form similar to (2), for which a solution for $A_{n}$ can be found from (7), the temperature distribution shown in figure $7 \mathrm{~b}$ and a reasonable value of $\epsilon$ that insures convergence of the resulting series.

Two of the objectives of the NBS Heat Transfer Laboratory are to develop and analyze methods for the determination of thermal conductivity of materials which vary widely in thermal properties and to make measurements of thermal conductivity for government agencies. For both analysis and experiment, the smoothing or bridging relationships described are applicable.

(Paper 71C2-248) 